médecine/sciences 1999; $15: 260-3$

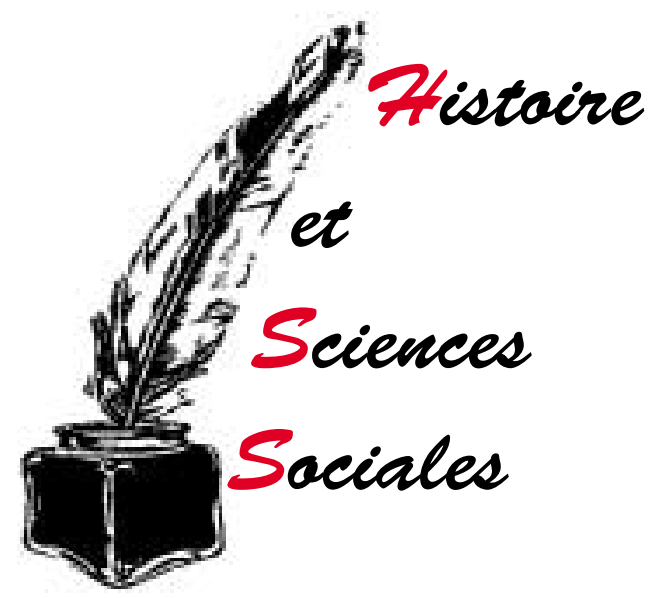

\title{
L'enfant endormi dans le ventre de sa mère
}

\section{La législation musulmane}

Toutes les sociétés éprouvent le besoin de légiférer sur la procréation. Qu'elle soit orale ou écrite, la loi fonde le social en désignant des pères, des fils, des héritiers, en créant des liens de parenté ainsi que les droits et les devoirs qui découlent de la place de chacun. La gestation est cernée par les lois: selon la date de naissance et les durées légales de grossesse admises, on peut présumer le moment de la conception; la filiation paternelle est susceptible d'être établie.

Les anciens juristes musulmans se sont longuement interrogés sur l'établissement d'une durée maximale de grossesse qui, alliée à la présomption de paternité bénéficiant au mari, indiquerait la voie à suivre pour résoudre le problème posé par la naissance d'un enfant dont la mère, au moment de l'accouchement, est veuve ou répudiée: qu'en sera-t-il de la légitimation de l'enfant né dans ces circonstances ? La femme accouchée sera-t-elle ou non accusée d'adultère, voire châtiée?

C'est ici que la durée maximale légale de grossesse intervient pour faire la part des choses et dire ce que sera le sort d'une femme et d'un enfant. Ainsi que nous le laissions entendre, le droit musulman a admis de longues durées de grossesse. Sur ce point particulier comme dans d'autres domaines, la doctrine juridique fut établie dès les premiers siècles de l'islam. Cependant, en la matière, elle est caractérisée par la pluralité des opinions: la durée s'étend de neuf mois à une durée illimitée en passant par plusieurs valeurs intermédiaires dont certaines - deux ans, quatre ans et cinq ans sont inconstablement orthodoxes, c'est-à-dire qu'elles correspondent à des opinions dominantes dans au moins une des écoles reconnues au moment où il a été considéré, vers le début du Iv ${ }^{\mathrm{e}}$ siècle de l'hégire, que le droit musulman était constitué et que devait cesser l'effort jurisprudentiel.

Cela passera peut-être pour un paradoxe, mais l'opinion selon laquelle la grossesse ne pouvait durer que neuf mois, n'est revendiquée que par l'école juridique dite "zahirite », école mineure par le nombre de ses adeptes et depuis longtemps disparue, et qui jamais ne réussit à faire admettre son point de vue à la majorité des musulmans. Au contraire, ce sont les longues durées qui ont prévalu dans les autres écoles, tant chez les hanéfites que chez les malékites, les chaféites et les hanbalites.

Pour la suite de notre approche juridique, nous nous limiterons à l'examen de la doctrine malékite, car c'est à elle que se réfèrent très largement, et depuis longtemps, les Maghrébins. Cette école tient son nom d'un juriste de Médine: l'imam Malik ben Anas. Malik naquit vers l'an 93 de l'hégire, soit en 712 de l'ère chrétienne et mourut en $179 / 796$. On lui te dans le temps à la grossesse, sinon celle décidée par Dieu. 
doit le Muwatta qui est un recueil de hadiths, et un volumineux ouvrage de droit, la Moudawwana I-koubra, dont il n'est pas directement l'auteur, mais qui fut écrit par ses disciples, en se fondant sur son enseignement, et qui lui est attribué.

La Moudawwana se présente comme un livre de droit dans lequel le disciple, ici Sahnoun, cadi de Kairouan, interroge le maître, Ibn al-Qasim, juriste égyptien, lequel connut à Médine Malik ben Anas. A même époque, les juristes de l'école hanéfite avaient défini la durée maximale légale de grossesse en se fondant sur un hadith attribué à Aïcha bint Abi Bakr, une épouse du Prophète. Selon eux, Aïcha avait dit: "L'enfant ne reste pas dans la matrice de sa mère plus de deux ans. » Reprenant cette durée limite du point de vue hanéfite, Sahnoun dans la Moudawwana interroge Ibn al-Qasim et lui demande:

- Qu'advient-il si un homme répudie sa femme et qu'elle accouche plus de deux ans après? L'enfant est-il oui ou non rattaché à l'époux?

Et Ibn al-Qasim de répondre:

- Il l'est, selon le dire de Malik, si elle accouche dans les trois, quatre ou cinq ans, et moi-même j'admets le délai de cinq ans. Malik disait: «On rattachera au mari l'enfant dont une femme accouche dans les délais semblables à ceux dont les femmes peuvent accoucher du fait de leur mari. » On remarquera que l'argumentation fait référence à un usage connu à Médine. Il va de soi que l'école malékite n'ignore ni le Coran ni la Sunna comme sources matérielles de droit. Cela dit, il convient d'observer que ce ne sont pas ces sources-là qui ont été sollicitées par les malékites pour justifier la doctrine mais des cas d'espèce attestant d'un usage établi. Dans le domaine médinois, en effet, les exemples ne manquent pas où des opinions favorables à l'admission de grossesses de longue durée s'affirment. Lorsque l'on rapporte à Malik les paroles attribuées à Aïcha et selon lesquelles la grossesse de la femme ne dure pas plus de deux ans, l'imam de Médine s'insurge:

- «Gloire à Dieu! Qui a pu dire cela? Vois notre voisine, la femme de Mahammad ben Ajlan, elle a porté son enfant quatre années avant d'accoucher. »

De cet enfant, on dira qu'à sa naissance, deux incisives lui étaient déjà poussées. D’un autre enfant, né également après avoir été longtemps porté, on remarquera qu'il avait beaucoup de cheveux, d'un autre encore, qu'il riait lors de l'accouchement. Les signes de la naissance sont là qui, interprétés, viennent parfois démontrer l'exceptionnelle maturité du nouveau-né: maturité physiologique de l'enfant qui naît muni d'une dentition ou coiffé d'une chevelure abondante, maturité psychologique de l'enfant qui rit dans des circonstances dans lesquelles les autres enfants ne font entendre que des pleurs et des cris, maturité qui vient accréditer l'idée qu'il y a bien longtemps que ce nouveau-né a été conçu. C'est ainsi que des cas personnels ont joué un rôle déterminant dans l'élaboration de la doctrine. Malik ben Anas lui-même était réputé avoir été porté deux ou trois années par sa mère - les textes anciens divergent sur ce point - et il est des savants musulmans pour penser que son propre exemple avait aussi influencé son choix juridique qui a fini par se cristalliser d'une manière quasiment intangible par la Moudawwana dans la doctrine malékite.

\section{L'aggiornamento juridique}

Intangible, la doctrine malékite le resta jusqu'à la période moderne.

Des dispositions, comme celles relatives à la durée de grossesse, s'intègrent dans un ensemble juridique que les musulmans nomment «Charia». La Charia, du point de vue de l'islam, est un ensemble de règles de droit qui sont, soit contenues dans le Coran, soit énoncées dans le hadith, soit établies par des juristes mais voulues par Dieu. La traduction du terme «Charia» par «Loi Divine», rend bien compte du phénomène idéologique qui sacralise un ensemble de règles et l'idée que la loi musulmane est divine a concouru au conservatisme : l'ensemble législatif concernant la famille musulmane s'est montré, à travers les siècles, particulièrement résistant au changement.
Mais ni le Maghreb ni la terre d'islam ne sont coupés du monde et, à partir de la seconde moitié du XIX ${ }^{\mathrm{e}}$ siècle, le contact de la science religieuse musulmane avec un savoir médical de plus en plus au fait des réalités physiologiques suscita des controverses. Une partie des élites maghrébines ne restait pas insensible à la découverte de connaissances nouvelles. Quand vint le moment des Indépendances, près de douze siècles s'étaient écoulés depuis la formation de l'ancienne école juridique de Médine. La colonisation était passée par la quasi totalité du monde musulman. Un savoir renouvelé avait gagné les facultés de médecine, de droit et même de théologie: c'est de la plus célèbre université musulmane, al-Azhar au Caire, qu'est issu Muhammad Abu Zahra, juriste contemporain d'obédience hanéfite, qui en termes pondérés en appelait aux nécessaires ajustements. Dans sa biographie de Malik ben Anas, il revint sur les circonstances entourant la naissance de l'imam de Médine.

A un homme exceptionnel, une naissance exceptionnelle, écrivait en substance le Cheikh Abu Zahra pour expliquer l'attachement des historiographes arabes à rappeler la longue durée pendant laquelle Malik aurait été porté par sa mère. Pour autant, Abu Zahra ne prenait plus cette assertion à son compte, pas davantage que les durées de grossesse légalisées par le droit musulman. Il écrivit: «... si Malik a une opinion juridique qui (...) s'inspire d'informations transmises par des mères ou des propos attribués à certaines femmes d'ancêtres connus pour leur honorabilité, nous, nous ne sommes pas en mesure de faire le même choix. » Abu Zahra choisissait d'opposer aux thèses classiques du droit musulman les connaissances scientifiques auxquelles il adhérait.

Cette manière de voir la grossesse s'est imposée dans les législations nationales aux responsables maghrébins, quelles qu'aient été par ailleurs leurs attitudes vis-à-vis d'autres dispositions du droit (répudiation, polygamie, règles de succession...). La Tunisie, dès l'indépendance en 1956, avait sauté le pas - un an - suivie par 
le Maroc qui adoptait lui aussi une année, tout en conservant une trace du droit classique par la porte laissée ouverte à des durées plus longues: à compter d'une année après sa répudiation ou le décès de son mari, la femme qui prétend être enceinte peut saisir le juge pour faire reconnaître que son état remonte à l'époque où elle était encore mariée. Le juge, en se fondant sur une expertise médicale, pourra accorder un délai à la femme pour accoucher... L'Algérie, enfin, optait dans son code de la famille (1984) pour une durée maximale de grossesse de dix mois.

Entrant dans le concert des nations, à côté de références plus ou moins marquées à l'islam, les États maghrébins étaient aussi soucieux de s'inscrire dans la modernité. L'un des prix à payer était l'abandon d'une disposition législative, certes originale et certainement utile, mais ne pouvant plus être admise sans réticence par une partie des populations qui n'aurait pas compris l'existence d'une telle dichotomie entre la physiologie et le droit.

\section{Les fonctions de la croyance}

En étudiant l'enfant endormi, notre présupposé de départ était que si cette croyance existe, si elle a conservé sa vitalité malgré l'évolution juridique et celles des connaissances scientifiques, c'est parce qu'elle a une utilité.

Le Dr Mouloud Lounaoussi, médecin en Kabylie qui avait bien voulu participer à un entretien pour mon travail de thèse*, est un spécialiste en gynécologie-obstétrique. Dans l'exercice de son art, il est confronté à l'autre savoir, celui de la tradition. Le domaine médical dont il est un spécialiste fut longtemps un domaine strictement réservé aux femmes. Son savoir qu'il tient des universités, ne peut oblitérer complètement l'autre savoir auquel il n'adhère pas, mais qui rentre en ligne de compte dans sa pratique comme élément de réfé-

* J. Colin, L'enfant endormi dans le ventre de sa mère, Étude ethnologique et juridique d'une croyance au Maghreb, CERJEMAF. Presses universitaires de Perpignan, 1998. Préface de C. Lacoste-Dujardin. rence pour ses patientes et leur famille.

A l'hôpital de Tizi Ouzou où le Dr M. Lounaoussi a travaillé au service de gynécologie-maternité, la question de l'enfant endormi revient fréquemment. Selon ce médecin, ce sont des «raisons sociales » qui expliquent cette fréquence. Ce qu'il a observé recoupe les fonctions de la croyance en l'enfant endormi telles que nous avons pu les rencontrer dans l'enquête de terrain et l'étude bibliographique.

- Cacher des relations sexuelles adultérines: ce cas de figure est quasiment un "classique" que l'immigration des hommes a largement favorisé. Devenir enceinte alors que le mari est absent, telle est la situation dans laquelle se sont retrouvées des femmes; faire remonter cette grossesse à l'époque du dernier retour au pays du mari, expliquer sa manifestation relativement tardive par le réveil d'un enfant endormi, puis faire admettre le tout par le mari et la belle-famille, tel est le parcours difficile qu'il leur a fallu accomplir pour traverser une période dangereuse.

Quand il s'agit d'adultère, explique le Dr M. Lounaoussi, la femme sait bien ce qu'elle dit, elle sait avec qui elle a conçu l'enfant mais elle ne l'avoue pas au médecin. En aucun cas, celui-ci ne peut obtenir une attitude franche de sa patiente. Ceci est bien normal car cet enfant endormi dont elle parle vient prendre la place de quelque chose - les conditions réelles dans lesquelles a été conçu l'enfant - dont la femme doit garder le secret sous peine de se condamner... peut-être à mort.

- Autre cas de figure, aux conséquences moins dramatiques mais grave tout de même: la stérilité. Dans une société où le rôle des femmes est de procréer, l'épouse qui ne met pas d'enfants au monde, est dans une situation que fragilisent le risque de la répudiation et les stigmates que peut porter à jamais celle qui a été répudiée pour une telle raison. Là encore, l'enfant endormi est invoqué. Plaçant la femme qui n'enfante pas quelque part entre la fertilité et la stérilité, c'est une demi-mesure qui peut se prolonger pendant plusieurs années dans l'attente de l'enfant espéré. Le fait d'avoir ses règles est certes un handicap pour la femme qui voudrait faire croire à une grossesse, mais il n'est pas insurmontable car l'on admet aussi qu'une femme puisse être enceinte sans aménorrhée: c'est une vision des choses connue au Maghreb dont on trouve un équivalent particulièrement bien exposé dans un hadith recensé par Malik ben Anas, où l'on voit une vieille femme expliquer à Umar, le second calife, que le sang des règles peut couler sur le foetus. Mais il est possible aussi que la femme manifeste des symptômes - aménorrhée, nausée, vomissement, ventre ballonné - et que, dans son désir d'enfant, elle se soit convaincue d'être enceinte alors qu'elle ne l'est pas. Cette dernière attitude concerne jusqu'à des femmes ménopausées qui n'arrivent pas à admettre que leur rythme physiologique a changé et qu'elles ont perdu leur fertilité d'antan.

Plus que l'ignorance des choses de la physiologie féminine, ce sont, à notre sens, ces «raisons sociales» évoquées par notre médecin, qui expliquent le mieux la vitalité de la croyance en l'enfant endormi. Que l'on pense, en particulier, à la question des relations sexuelles hors mariage. La société et le droit ont cela de commun qu'ils enregistrent des attitudes extrêmes face à des actes sexuels illicites : la société pousse au crime d'honneur tandis que le droit religieux requiert l'application de châtiments parmi lesquels la flagellation ou la lapidation sont prescrites contre l'adultère.

De telles attitudes n'ont pas été sans poser des problèmes, car à s'obliger à l'application de peines extrêmes, on finit par se mettre dans des situations dont les défauts sont évidents. Ni les sentiments de honte et d'honneur, ni les devoirs à l'égard de Dieu n'ont réussi à rendre simple et automatique l'application de telles sanctions. «Il peut être difficile pour un père de passer le couteau», disait le Dr Lounaoussi. Ce problème survient avec toute loi trop sévère dont l'observation scrupuleuse pourrait être pire que le mal qu'elle voudrait combattre. La situation cependant, n'est 
pas sans issue: tout se passe comme si des modalités contradictoires traversaient la société et le droit de telle sorte qu'au rigorisme violent et excessif qui se manifeste dans le crime d'honneur et les châtiments légaux, s'oppose un antidote porteur de détente, l'enfant endormi et les longues durées de grossesse, capable de rétablir l'ordre lui aussi, mais par des solutions apaisantes.

On peut comprendre qu'au nom de la modernité, la classe dirigeante des pays maghrébins ait voulu réformer le droit. Mais si la société continue de voir dans l'enfant né de relations sexuelles hors mariage, un bâtard, dans l'homme et la femme qui ont ces relations, des débauchés, elle n'a alors parcouru que la partie la plus facile du chemin

\section{Joël Colin}

3, rue de Bruxelles, 67000 Strasbourg, France.

\section{Summary}

The child asleep in its mother's bosom

Muslim law, having originated in the Orient, institutionalized local customs in the course of its subsequent development. Taking into account the traditions of Medina, and without explicit reference to the Koran or the Sunna, particularly Malekism allowed for pregnancies of up to four or five years.

Islamisation of the Maghreb brought such legal tendancies into contact with native Berber culture. Field surveys reveal a belief in North Africa according to which during pregnancy the human foetus can stop its development and remain in a woman's womb until her death, or successivly resume growth and be born. A widespread image of such a belief is that of a child asleep in its mother's bosom. From this legal-cultural junction results a certain consensus, which Maghrebin legists have recorded by admitting gestation periods beyond limits initially fixed. Analysis of such views, recorded in texts as well as in field work, suggests advantages which can be drawn from them by the given populations, particularly concerning questions of filiation.
TIRÉS À PART

J. Colin.

\section{LIGR}

Enseignement organisé dans le cadre de la Faculté de Médecine Paris-Sud, et de l'Université Paris XI.

- Début : 3 novembre 1999

- Enseignement de 2 ans à temps plein, destiné aux jeunes cancérologues diplômés, titulaires par ailleurs d'un DEA ou équivalent, et désireux de recevoir un enseignement de haut niveau en recherche clinique en cancérologie. Ouvert aux spécialistes, Docteurs en médecine, Oncologues médicaux et pédiatres, Chirurgiens, Radiothérapeutes, Radiologues, Pathologistes, Biologistes, Statisticiens, etc., et aux Docteurs en Pharmacie.

- De toutes nationalités, mais une très bonne connaissance du français et de l'anglais est indispensable. Cours en Français, certains exposés et mémoires pourront être présentés en Anglais.

- Une formation approfondie dans le domaine de la recherche clinique en cancérologie est offerte, comportant un enseignement théorique, et des stages cliniques ou de laboratoire.

> Fonctions effectives avec responsabilités pendant 2 ans à plein temps, dans sa spécialité d'origine, et 6 mois au minimum dans une autre, à l'institut GustaveROUSSY. Option : 1 année sur les 2 consacrée à un travail personnel dans un laboratoire de recherche de l'IGR.

$>$ Enseignement théorique, obligatoire et commun à tous, de 340 heures en 2 ans. Il comporte : 8 modules de 5 jours (cours, discussions de dossiers, de documents ou de techniques, avec forte participation des élèves), les uns généraux, les autres spécialisés : 1 enseignement de statistiques, épidémiologie et santé publique réparti sur les 2 ans ; 1 séminaire de 2 à $3 \mathrm{~h}$ tous les 15 jours sur un sujet limité.

$>$ Travail personnel de recherche clinique ou biologique aboutissant en 2 ans à la rédaction et la soutenance de 1 ou 2 mémoires.

$>$ Formation individualisée, reposant sur un encadrement très proche : tuteur, directeur de stage et de mémoire.

- L'attribution du Diplôme fait suite à une évaluation finale portant sur les notes des examens suivant chaque module, l'évaluation des mémoires, l'avis du « tuteur » de chaque étudiant et celui des responsables de ses stages.

- Les promotions sont limitées à 15 élèves chaque année.

- Les candidats pourront éventuellement bénéficier d'une bourse ou d'un salaire de 2000 Euros par mois pour leur première année (\# 2350 \$ US). Ils sont invités à se procurer une bourse pour la $2^{e}$ année.

Renseignements et dossier de candidature à demander à Mme Anne-Marie RIVIÈRE par courrier ou E-mail : arivière@igr.fr. Candidatures par écrit avant le $1^{\text {er }}$ avril 1999, adressées au Professeur Jean LEMERLE, Directeur du D.U.E.R.C.C. (E-mail : lemerle@igr.fr.) ou au Professeur Martin SCHLUMBERGER, Directeur des Études

(E-mail : schlumbg@igr.fr) - INSTITUT GUSTAVE-ROUSSY, 94805 VILLEJUIF (France) 